\title{
Profiling Circulating Inflammation and Microbial Translocation Biomarkers Associated with Advanced Liver Fibrosis in HIV/HCV Coinfected Persons
}

\section{Xiaochen Chen}

Fudan University School of Public Health

Xing Liu

Fudan University School of Public Health

\section{Song Duan}

Dehong Prefecture CDC

Renhai Tang

Dehong Prefecture CDC

\section{Sujuan Zhou}

Fudan University School of Public Health

\section{Runhua Ye}

Dehong Prefecture CDC

\section{Yuecheng Yang}

Dehong Prefecture CDC

Jibao Wang

Dehong Prefecture CDC

\section{Shitang Yao}

Dehong Prefecture CDC

$\mathrm{NaHe}$ ( $\nabla$ nhe@shmu.edu.cn )

Fudan University School of Public Health

\section{Research}

Keywords: HIV, HCV, liver fibrosis, microbial translocation, inflammation

Posted Date: April 6th, 2020

DOl: https://doi.org/10.21203/rs.3.rs-20121/v1

License: (c) (i) This work is licensed under a Creative Commons Attribution 4.0 International License. Read Full License 
Page $2 / 18$ 


\section{Abstract}

Background: HIV/HCV coinfection leads to accelerated liver fibrosis, in which microbial translocation and systemic inflammation might play important roles. This study aimed to provide an extensive profile of plasma microbial translocation and inflammation biomarkers associated with advanced liver fibrosis among HIV/HCV coinfected patients.

Methods:This cross-sectional study recruited $343 \mathrm{HIV} / \mathrm{HCV}$ coinfected patients on combination antiretroviral therapy (cART) from a rural prefecture of Yunnan province in Southwest China. Plasma concentrations of SCD14 and 27 cytokines/chemokines were assayed, and were compared against advanced or mild levels of liver fibrosis.

Results: Of the $343 \mathrm{HIV} / \mathrm{HCV}$ coinfected patients, 188 (54.8\%) had severe or advanced liver fibrosis (FIB-4 $>3.25$ ). Patients with advanced liver fibrosis (FIB-4 $>3.25$ vs. FIB- $4 \leq 3.25$ ) had higher plasma levels of IL1 $\beta$, IL-6, IL-7, IL-9, IL-12, IL-15, IL-17, GM-CSF, IFN- $y$, TNF-a, IL-4, IL-10, IL-13, FGF-basic and MCP-1. Multivariable logistic regression models showed that advanced liver fibrosis was associated with increased plasma level of IL-1 $\beta$, IL-6, IL-7, IL-12, IL-17, GM- CSF, IFN- $\gamma$, IL-4, IL -10, MCP-1, Eotaxin and FGFbasic, with FGF-basic remained to be positively and significantly associated with advanced liver fibrosis after Bonferroni correction for multiple comparisons ( $\mathrm{aOR}=1.82 ; 95 \% \mathrm{Cl}: 1.26-2.66 ; p=0.002$ ). Plasma sCD14 was also significantly associated with advanced liver fibrosis (aOR=1.14; 95\%Cl: 1.01-1.30; $p=0.048)$.

Conclusions: HIV/HCV coinfected patients are living with high prevalence of advanced liver fibrosis which coexists with a mixture of elevated plasma inflammation and microbial translocation biomarkers. The significant associations of advanced liver fibrosis with FGF-basic and SCD14 may reveal pathogenic mechanisms and potential clinical intervention targets of liver fibrosis in HCV/HIV coinfection.

\section{Background}

Coinfection with human immunodeficiency virus (HIV) and hepatitis $\mathrm{C}$ virus (HCV) brings heavy disease burden to the infected population, and is a major public health concern worldwide[1]. HIV/HCV coinfected patients have more frequent and accelerated progression to fibrosis and end-stage liver diseases (ESLD) including cirrhosis and hepatocellular carcinoma (HCC) compared to HCV monoinfected patients[2]. This is a serious public health challenge in China given the fact that China has a large number of HIV/HCV coinfected patients who were most likely to be former plasma donors or injection drug users[3]. However, different from western countries, direct-acting antivirals (DAAs) or even peg-interferon plus ribavirin (PR) for HCV treatment are not widely used in China especially in rural areas because of limited access to health care and high prices of the drugs, which leads to a heavier disease burden[4]. Pathogenesis of progressive liver fibrosis in HIV/HCV coinfection is complex[5], and is mainly characterized by higher HCV viral load, altered cellular immunity, elevated pro-inflammatory and profibrogenic cytokines. More specifically, the immune suppression and CD4 T-cell depletion induced by HIV infection cause persistent 
immune activation, and Kupffer cells and hepatic stellate cells (HSCs) are directly stimulated to secrete pro-fibrotic cytokines or type 1 collagen[6]. In addition, HIV infection causes weakened CD4 and CD8 T cell responses, leading to the persistence of HCV infection [7], which in turn contributes to the increased rates of liver fibrosis[8].

Moreover, microbial translocation (MT), featured by dysfunction of mucosal barrier and increased intestinal permeability due to immune dysregulation and intestinal microbiome change, has been commonly observed among HIV-infected individuals[9]. Microbial components including, lipopolysaccharide (LPS) \lipoteichoic acid and flagellin may promote liver fibrosis by stimulation of HSCs and/or Kupffer cells with LPS binding protein (LBP) and soluble CD14 (sCD14). These microbial components may enhance hepatic inflammation, immune response and liver cell death[5].

Thus, the above-mentioned mechanisms jointly emphasize the important role played by inflammation in liver fibrosis among HIV/HCV coinfected patients. Cirrhosis-associated dysregulation of immune responses can be reflected by increased production and elevated circulatinglevels of pro-inflammatory cytokines[10]. Kupffer cells generate IL-1b, IL-6, IL-12, IL-18 and also release anti-inflammatory cytokines, including IL-10 stimulated by LPS[11]. Upregulation of IL-4, IL-13, TGF-b1 and platelet-derived growth factor (PDGF) was observed during fibrogenesis, while gp120 induces HSC accumulation by secretion of monocyte chemoattractant protein-1 (MCP-1) by HSCs among HIV-infected patients[12]. Furthermore, HIV suppression by combination antiretroviral therapy (cART) may decrease inflammation and immune activation, and may slow down the progression of liver disease[13], however, the levels of plasma inflammatory biomarkers may remain abnormal in many individuals[14]. So far, the inflammatory profile among HIV/HCV coinfected patients with different levels of liver fibrosis has not been well established, especially under the circumstance of combination antiretroviral therapy (cART). To fill this gap, the present study aimed to examine the association of advanced liver fibrosis with microbial translocation and inflammation cytokines among HIV/HCV coinfected patients in the era of CART.

\section{Materials And Methods}

\section{Study sample}

The present cross-sectional study was conducted in Dehong Prefecture of Yunnan Province at China's southwest border, with injection drug use (IDU) as the predominant mode of HIV transmission through the early 2000s and continues to be an important mode of HIV and HCV infection[15]. By March 2016, 1,017 HIV/HCV coinfected patients with 170 (16.7\%) deaths had been reported to and registered in the Comprehensive Response Information Management System (CRIMS) for HIV/AIDS in China[16]. Out of the remaining 847 patients, $463(54.7 \%)$ were identifiable during the study period from April to October in 2016. Of them, 390 (84.2\%) were receiving cART and gave informed consent to participate in the present

study. Forty-seven patients were further excluded from the study due to missing data on biochemical measures for FIB-4 calculation including aspartate aminotransferase (AST), alanine aminotransferase (ALT), and platelet count (PLT). Thus, a total of $343 \mathrm{HIV} / \mathrm{HCV}$ coinfected patients were included in the 
final analysis. These participants showed no significant differences with the 120 identified yet excluded patients in the distribution of age, sex, marital status, HIV transmission route, HBsAg serostatus, baseline CD4 cells count, years on CART, current HCV RNA and HIV RNA levels, while showed difference in ethnicity (Table S1). The study was approved by the Institutional Review Board of Fudan University School of Public Health, Shanghai, China. All subjects provided informed consent prior to enrollment.

\section{Data Extraction}

Demographical and clinical epidemiological data were extracted from the CRIMS. The data included age, sex, marital status, ethnicity, HIV transmission route, date of cART initiation, CART regimen, CD4+ T cell counts at cART initiation and follow-up visits.

\section{Blood testing}

Biochemical tests for liver fibrosis assessment

Liver biopsy is an invasive technique with complications and costly, though it is a gold reference standard for determining liver fibrosis. In the light of these limitations, we assessed liver fibrosis by FIB-4 score, calculated as (AST [IU/L] $\times$ age [years]) / (PLT [10\% $/ \mathrm{L}] \times$ ALT [IU/L] $\left.]^{1 / 2}\right)$ using Sterling's formula [17], which is an internationally recognized well-established noninvasive indicator for liver fibrosis[18]. Biochemical measurements of AST, ALT and PLT were performed using an automatic biochemistry analyzer (Beckman Coultner, USA), according to the manufacturer's protocol.

FIB-4 is generally divided into three categories: no or mild liver fibrosis with FIB-4 $<1.45$, intermediate liver fibrosis with $1.45 \leq$ FIB-4 $\leq 3.25$, and advanced liver Fibrosis with FIB-4 $>3.25$. In this study, we considered advanced liver fibrosis (FIB-4 $>3.25$ ) as the major outcome.

\section{HCV RNA Quantification and genotyping}

Plasma HCV viral RNA was extracted (Roche diagnostic products (Shanghai) Co., Ltd., China), and quantified by a real-time polymerase chain reaction (RT-PCR) technique using commercially available kits for the quantification of HCV RNA (PCR-Fluorescent Probing, PG Biotech Ltd., Shenzhen, China). The limit of detection was 500 copies $/ \mathrm{ml}$, and the linear range of HCV RNA quantification was from $1.0 \times 10^{3}$ to $5.0 \times 10^{7}$ copies $/ \mathrm{ml}$.

Amplification was completed by a nested PCR with E1- or NS5B-specific primers. Splicing, proofreading, and aligning sample sequences were performed using ChromasPro 1.5 and BioEdit7.0.9.0 software. HCV genotype reference sequences were retrieved from the HCV database (http://hcv.lanl.gov/content/sequence/HCV/ToolsOutline.html). The HCV gene subtype was determined according to the phylogenetic tree,which established by the Neighbor-joining method using MEGA 7.0 software[19]. 
Twenty-seven cytokines, chemokines and growth factors cytokines in plasma specimens were quantified by a multiplex analysis performed on the BioPlex ${ }^{\circledR} 200$ Multiplex System platform using the Bio-Plex Human 27-plex panel of cytokines/chemokines/growth factors (Bio-Rad, Hercules, CA, USA), according to the manufacturer's instructions. These biomarkers included 1) proinflammatory biomarkers such as interleukin (IL)-1 $\beta$, IL-2, IL-6, IL-7, IL-8, IL-9, IL-12, IL-15, IL-17, granulocyte colony stimulating factor (G$\mathrm{CSF}$ ), granulocyte macrophage colony stimulating factor (GM-CSF), Interferon- $\gamma(\mathrm{IFN}-\mathrm{\gamma})$, and tumor necrosis factor (TNF-a); 2) anti-inflammatory biomarkers such as IL-1ra, IL-4, IL-5, IL-10, and IL-13; 3) chemokines such as IFN- $\gamma$-inducible protein (IP-10), monocyte chemoattractant protein-1 (MCP-1), macrophage inflammatory proteins-1alpha (MIP-1a), MIP-1b, and regulated upon activation normal T-cell expressed and secreted (RANTES); and 4) growth factors such as Eotaxin, fibroblast growth factor 2 (FGF-basic), platelet-derived growth factor (PDGF-BB), and vascular endothelial growth factor (VEGF).

The detection limit for each molecule was determined by the recovery of the corresponding standard, and the lowest values with more than $70 \%$ recovery were set as the lowest detection limits. Measurements less than the lower limit of quantification (LLOQ) were assigned a value of half the LLOQ, and measurements more than the upper limit of quantification (ULOQ) were assigned a value of twice the ULOQ for each marker.

Plasma sCD14 was measured according to the manufacturer's protocol for commercial enzyme-linked immunosorbent assay (ELISA) (Hycult Biotech, Wayne PA, USA).

\section{Other tests}

Human hepatitis B virus surface antigen (HBsAg) was tested by ELISA technique (Wan Tai Biomedical Co. Ltd, Beijing, China). CD 4 cell counts were assessed by FACSCount (Becton, Dickinson and Co., San Jose, CA, USA).

\section{Statistical analysis}

Distributions of categorical variables were compared using chi-square test or Fisher's exact test, and continuous varabiles were compared using t-test or Mann-Whitney $U$ test. Log10-transformation was conducted for plasma cytokines levels for further statistical and regression analyses. A multiple logistic regression analysis with adjustment for age (as a contiuous variable), sex (0-male, 1-female), ethnicity (0Han, 1-Dai, 2-Jingpo,3-Others, entered as dummy variables), current HIV RNA (0-Undetectable, 1-

Detectable), current HCV RNA (0-Undetectable, 1-Detectable), current CD 4 cell counts $((0-<200,1-200-349$, $2-\geq 350$, entered as dummy variables), years on CART (as a contiuous variable) and ART regimen type ((0NVP, 1-TDF, 2-Others, entered as dummy variables) was used to explore the association of liver fibrosis with the plasma level of each of the twenty-seven cytokines and SCD14. The odds ratio (OR) and 95\% confidence interval $(95 \% \mathrm{Cl})$ represent the risk of higher FIB-4 with per one log-unit change in plasma cytokine concentration. Spearman correlations were computed to explore correlations between the plasma inflammatory biomarkers. Statistical significance was defined as $P<0.05$ and Bonferroni $P$ $<0.002(0.05 / 27 \approx 0.002)$ for multiple comparison adjustment. All statistical analyses were performed using R software (version 3.3.2). 


\section{Results}

\section{Demographic and clinical characteristics}

The median age of the participants was 35.4 (IQR: 31.3-39.3) years old. The majority of the participants were male (98\%), had injection drug use (IDU) as HIV transmission route (91.5\%), seronegative for hepatitis B surface antigen (HBsAg) (91.3\%), detectable for plasma HCV RNA (69.4\%) but undetectable for plasma HIV RNA (89.3\%). Around half of the participants were non-Han ethnicities, i.e., ethnic minorities (54.2\%), currently married (51.9\%), and of HCV subtype 3 (47\%) (Table 1 ).

\section{Prevalence of Advanced Liver fibrosis}

Among all participants, 75 (21.9\%) had no or mild liver fibrosis (FIB-4), 80 (23.3\%) had intermediate liver fibrosis (FIB-4), and 188 (54.8\%) had severe or advanced liver fibrosis (FIB-4 >3.25). Compared to patients without advanced liver fibrosis, those with advanced liver fibrosis were older, under different CART regimen, and had longer duration on cART (Table 1).

Table 1. Demographic and clinical characteristics of HIV/HCV coinfected participants 


\begin{tabular}{|c|c|c|c|c|}
\hline & \multirow{3}{*}{$\begin{array}{c}\text { All } \\
\text { No. (\%) }\end{array}$} & \multicolumn{2}{|c|}{ FIB-4 score } & \multirow{3}{*}{$p^{*}$} \\
\hline & & $\leq 3.25$ & $\square 3.25$ & \\
\hline & & No. (\%) & No. (\%) & \\
\hline Overall & $343(100.0)$ & $155(45.2)$ & $188(54.8)$ & \\
\hline \multicolumn{5}{|l|}{ Age, years } \\
\hline Median (IQR) & $35.4(31.3-39.3)$ & $34.6(30.2-38.6)$ & $36.0(31.8-40.3)$ & 0.029 \\
\hline \multicolumn{5}{|l|}{ Sex } \\
\hline Male & $336(98.0)$ & $151(97.4)$ & $185(98.4)$ & 0.796 \\
\hline Female & $7(2.0)$ & $4(2.6)$ & $3(1.6)$ & \\
\hline Marital status & & & & 0.210 \\
\hline Unmarried & $130(37.9)$ & $61(39.4)$ & $69(36.7)$ & \\
\hline Married & $178(51.9)$ & $74(47.7)$ & $104(55.3)$ & \\
\hline Divorced/widowed & $35(10.2)$ & $20(12.9)$ & $15(8.0)$ & \\
\hline Ethnicity & & & & 0.151 \\
\hline Han & $157(45.8)$ & $62(40.0)$ & $95(50.5)$ & \\
\hline Dai & $112(32.6)$ & $60(38.7)$ & $52(27.7)$ & \\
\hline Jingpo & $62(18.1)$ & $28(18.1)$ & $34(18.1)$ & \\
\hline Others & $12(3.5)$ & $5(3.2)$ & 7 (3.7) & \\
\hline HIV transmission route & & & & 0.185 \\
\hline IDU & $314(91.5)$ & $140(90.3)$ & $174(92.6)$ & \\
\hline Others & $29(8.5)$ & $15(9.7)$ & $14(7.4)$ & \\
\hline Baseline CD4, cells/ul & & & & 0.618 \\
\hline$<200$ & $98(29.0)$ & $40(26.3)$ & $58(31.2)$ & \\
\hline $200-349$ & $139(41.1)$ & $65(42.8)$ & $74(39.8)$ & \\
\hline$\geq 350$ & $101(29.9)$ & $47(30.9)$ & $54(29.0)$ & \\
\hline Current CD4, cells/ul & & & & 0.511 \\
\hline$<200$ & $21(6.1)$ & $7(4.5)$ & $14(7.4)$ & \\
\hline $200-349$ & $52(15.2)$ & $23(14.8)$ & $29(15.4)$ & \\
\hline$\geq 350$ & $270(78.7)$ & $125(80.6)$ & $145(77.1)$ & \\
\hline HCV RNA & & & & 0.234 \\
\hline Undetectable & $105(30.6)$ & $53(34.2)$ & $52(27.7)$ & \\
\hline Detectable & $238(69.4)$ & $102(65.8)$ & $136(72.3)$ & \\
\hline HIV RNA & & & & 0.053 \\
\hline Undetectable & $264(89.3)$ & $126(88.1)$ & $138(79.3)$ & \\
\hline Detectable & $53(10.7)$ & $17(11.9)$ & $36(20.7)$ & \\
\hline Years on cART & $5.7(4.2-8.4)$ & $5.1(3.9-7.6)$ & $6.1(4.4-8.9)$ & 0.001 \\
\hline HBsAg positive & & & & 0.685 \\
\hline Yes & $30(8.7)$ & $12(7.7)$ & $18(9.6)$ & \\
\hline No & $313(91.3)$ & $143(92.3)$ & $170(90.4)$ & \\
\hline ART regimen, \% & & & & 0.012 \\
\hline NVP (vs. EFV/RTV) & $136(39.7)$ & $52(33.5)$ & $84(44.7)$ & \\
\hline TDF (vs. AZT/d4T/DDI) & $147(42.9)$ & $80(51.6)$ & $67(35.6)$ & \\
\hline Others & $60(17.4)$ & $23(14.8)$ & 37 (19.7) & \\
\hline HCV genotype & & & & 0.182 \\
\hline 1 & $46(16.0)$ & $23(18.1)$ & $23(14.4)$ & \\
\hline
\end{tabular}


aIDU, injection drug use;

${ }^{\mathrm{b}}$ Chi-square tests or Mann-Whitney $U$ test were performed wherever appropriate;

${ }^{\mathrm{C} B o l d}$ signifies statistical significance as $\mathrm{p}<0.05$

\section{Plasma levels of inflammatory cytokines}

Participants with sever liver fibrosis (FIB-4 >3.25) had significantly higher plasma levels of IL-1 $\beta$, IL-4, IL-6, IL-7, IL-9, IL-10, IL-12, IL-13, IL-15, IL-17, IFN-y, TNF-a, MCP-1, GM-CSF, FGF-basic and VEGF than those with FIB-4 $\leq 3.25$ (all $p<0.05$ ) (Table 2 and Figure 1). Such differences remained significant for IL-6, IL-10, IFN- $\gamma$, GM-CSF and FGF-basic after Bonferroni correction for multiple comparisons (all $p \leq 0.002$ or $0.05 / 27)$. No significant difference in SCD14 level was observed between the two groups.

\section{Table 2. Plasma level of inflammatory cytokines according to FIB-4 score}




\section{$\leq 3.25 \quad \square 3.25$}

\begin{tabular}{|c|c|c|c|c|}
\hline \multicolumn{5}{|c|}{ Pro-inflammatory } \\
\hline IL-1ß & $9.8(5.0,46.9)$ & $8.0(4.6,33.8)$ & $11.3(5.2,63.0)$ & 0.005 \\
\hline IL-2 & $6.9(0.50,87.0)$ & $6.1(0.5,63.4)$ & $9.6(0.5,95.6)$ & 0.216 \\
\hline IL-6 & $19.8(6.8,46.3)$ & $16.3(4.8,37.0)$ & $23.6(10.2,54.6)$ & 0.001 \\
\hline IL-7 & $14.4(6.2,29.8)$ & $13.2(5.3,24.6)$ & $15.6(6.4,35.0)$ & 0.038 \\
\hline IL-8 & $63.6(32.5,123.9)$ & $56.0(31.7,117.5)$ & $73.0(34.5,123.8)$ & 0.166 \\
\hline IL-9 & $92.8(39.4,267.5)$ & $81.5(32.7,183.4)$ & $103.0(50.0,337.1)$ & 0.009 \\
\hline IL-12 & $27.0(1.9,73.0)$ & $20.9(1.5,58.3)$ & $32.4(3.7,93.5)$ & 0.006 \\
\hline IL-15 & $23.0(5.3,112.1)$ & $14.8(5.3,86.4)$ & $27.6(5.3,127.0)$ & 0.046 \\
\hline IL-17 & $92.2(11.1,146.6)$ & $46.0(9.7,129.0)$ & $106.3(13.8,154.1)$ & 0.016 \\
\hline G-CSF & $52.0(27.2-82.0)$ & $44.5(20.7,89.7)$ & $54.8(36.1,80.1)$ & 0.278 \\
\hline GM-CSF & $93.7(0.3,238.3)$ & $17.6(0.2,196.2)$ & $121.0(2.2,269.3)$ & $<0.001$ \\
\hline IFN- $\gamma$ & $41.0(13.8,72.4)$ & $29.9(5.0,65.7)$ & $47.0(26.9,75.2)$ & 0.002 \\
\hline TNF- $\alpha$ & $46.0(31.1,70.0)$ & $40.8(27.1,70.3)$ & $50.5(35.6,68.3)$ & 0.015 \\
\hline \multicolumn{5}{|c|}{ Anti-inflammatory } \\
\hline IL-1r $\alpha$ & $324.5(141.0,755.4)$ & $288.9(124.1,722.8)$ & $338.1(156.0,801.7)$ & 0.210 \\
\hline IL-4 & $5.6(2.9,61.0)$ & $5.1(2.6,11.2)$ & $5.9(3.3,80.0)$ & 0.008 \\
\hline IL-5 & $10.0(4.5,22.0)$ & $10.6(4.3,20.0)$ & $10.0(4.7,23.0)$ & 0.632 \\
\hline IL-10 & $9.9(0.7,61.0)$ & $7.4(0.7,40.8)$ & $11.8(0.7,71.6)$ & 0.001 \\
\hline IL-13 & $8.4(3.0,32.0)$ & $6.6(2.1,20.9)$ & $10.1(3.7,34.1)$ & 0.006 \\
\hline \multicolumn{5}{|c|}{ Chemoattractant } \\
\hline IP-10 & $2390.3(1331.0,4196.2)$ & $\begin{array}{c}2322.5(1244.2 \\
4004.6)\end{array}$ & $2565.7(1492.8,4208.3)$ & 0.335 \\
\hline MCP-1 & $46.1(14.9,88.2)$ & $32.9(14.2,77.0)$ & $53.2(16.0,112.9)$ & 0.013 \\
\hline MIP-1 $\alpha$ & $4.9(3.0,37.0)$ & $5.1(2.6,19.2)$ & $4.8(3.4,42.3)$ & 0.102 \\
\hline MIP-1 $\beta$ & $132.2(84.1,410.5)$ & $126.0(87.6,235.9)$ & $142.5(80.4,551.3)$ & 0.229 \\
\hline RANTES & $\begin{array}{c}19871.0(11759.0 \\
69279.6)\end{array}$ & $\begin{array}{c}19700.5(9854.2 \\
69279.6)\end{array}$ & $\begin{array}{c}19979.5(13163.8 \\
69279.6)\end{array}$ & 0.881 \\
\hline \multicolumn{5}{|c|}{ Growth factor } \\
\hline Eotaxin & $182.7(117.7,294.1)$ & $179.1(117.6,263.7)$ & $193.0(118.6,324.2)$ & 0.126 \\
\hline $\begin{array}{l}\text { FGF- } \\
\text { basic }\end{array}$ & $66.0(9.8,104.5)$ & $40.9(4.35,97.1)$ & $79.5(30.6,107.0)$ & 0.001 \\
\hline $\begin{array}{l}\text { PDGF- } \\
\text { BB }\end{array}$ & $2840.7(1266.9,4561.9)$ & $\begin{array}{c}2750.9(1323.52, \\
4295.5)\end{array}$ & $2924.1(1231.3,4616.9)$ & 0.978 \\
\hline VEGF & $105.0(27.05,215.1)$ & $94.0(7.6,196.9)$ & $111.7(47.0,230.5)$ & 0.050 \\
\hline \multicolumn{5}{|c|}{ Microbial Translocation } \\
\hline sCD14 & $3.0(2.2,4.2)$ & $2.9(2.2,3.9)$ & $3.2(2.2,4.7)$ & 0.094 \\
\hline
\end{tabular}

Data were presented as median and interquartile range (IQR), with $\mu \mathrm{g} / \mathrm{mL}$ as the unit for SCD14 and $\mathrm{pg} / \mathrm{mL}$ for all other cytokines. All the p-values were calculated by Mann-Whitney tests.

\section{Correlations of inflammatory cytokines with advanced liver fibrosis}

Multivariable logistic regression adjusting for potential confounders was performed to examine the association of advanced liver fibrosis with each of the 27 cytokines/chemokines and SCD14 levels. 
Advanced liver fibrosis was shown to be associated with higher levels of eleven out of the twenty-seven markers $(p<0.05)$, while FGF-basic remained to be positively and significantly associated with advanced liver fibrosis after Bonferroni correction for multiple comparisons (aOR=1.82; 95\% Cl: 1.26-2.66; $p=0.002)$. Furthermore, plasma sCD14 was significantly associated with advanced liver fibrosis $(a O R=1.14 ; 95 \% \mathrm{Cl}$ : 1.01-1.30; $p=0.048$. Figure 2).

\section{Discussion}

Liver complications including liver fibrosis, cirrhosis and ESLD have emerged as an important concern of non-AIDS-defining morbidity and mortality among PLWH in China, especially among those who are coinfected with HCV and/or hepatitis B virus (HBV) $[16,20,21]$. In the present study, $54.8 \%$ of HIV/HCV coinfected patients had advanced liver fibrosis. The prevalence was much higher than studies conducted in western countries which ranged from $22.9 \%$ to $40.3 \%$ [22-24]. The discrepancy is possibly attributed to the fact that these the patients in our study are lack of antiviral therapy for viral hepatitis $C$ and have a high rate of heavy alcohol use[25].

HIV and HCV infections are both characterized by systemic inflammation with increased levels of blood inflammatory cytokines. The pattern of cytokine release may have been altered and immune-mediated inflammatory responses be exacerbated by HIV infection[26]. Although cART might help reduce plasma inflammatory marker levels in HIV/HCV coinfected patients, the markers may still remain much higher than healthy controls[27]. In the present study, we identified a mixed profile of elevated circulating inflammatory cytokines, chemokines and grow factors including IL-6, IL-10, IFN- $y$, GM-CSF and FGF-basic among HIV/HCV coinfected patients with advanced liver fibrosis. This finding further indicates that progressive and advanced liver fibrosis in HIV/HCV coinfected patients is mainly an inflammatory syndrome[12].

In multiple logistic regression analysis adjusting for potential confounders, FGF-basic was shown to be significantly and positively associated with advanced liver fibrosis upon Bonferroni correction for multiple comparisons. FGF-basic or Fibroblast Growth Factor-basic (FGF-basic/FGF-2/FGF- $\beta$ ) is a single-chain polypeptide growth factor that plays a significant role in the process of wound healing, is a potent inducer of angiogenesis, and has potent heparin-binding activity. Recent studies have suggested potential yet controversical associations of FGF-basic with disease status among HIV patients[28, 29]. The present finding of the positive association between FGF-basic and advance liver fibrosis may reveal a pathogenic mechanism and a potential clinical intervention target of liver fibrosis in HCV/HIV coinfection.

The significant associations of advanced liver fibrosis with higher levels of IL-6, IFN- $\gamma$, IL-10 and GM-CSF, despite unsignificant upon Bonferroni correction, are in concordance with some previous studies and worthy of further investigation [30-33] . IL-6 is profibrogenic and may induce liver damage[34]. The role of IFN- $\gamma$ in liver fibrosis is controversial. In some studies IFN- $\gamma$ was negatively correlated with liver fibrosis [35], while IFN- $\gamma$ was also found to have proinflammatory effects that can aggravate disease progression and organ dysfunction[32], and increased production of IFN- $\gamma$ is observed among cirrhotic patients[36]. 
Soluable IL-10 was higher in HIV/HCV coinfected patients than in HIV-negative persons[29], but could be reduced following peg-interferon plus ribavirin (PR) treatment for HCV[37]. GM-CSF was higer in HIV patients than in health control subjects[33], and may promote liver damage and liver fibrosis [38]. SCD14 is often employed as an indirect biomarker of microbial translocation, given the technical difficulty in measuring lipopolysaccharide (LPS) which is a major marker for microbial translocation [39]. Our study shows that SCD14 level was positively associated with advanced liver fibrosis, consistent with the previous studies [40,41], further suggesting the role of inflammation in liver complications in HIV/HCV patients.

This study has several limitations. First, no causal relationship could be drawn upon the cross-sectional design of this study. Second, data are unavailable on participants' alcohol drinking which is known to influence the risk of liver fibrosis and could alter levels of immune/inflammatory markers. This should be addressed in future research.

\section{Conclusions}

In summary, HIV/HCV coinfected patients are living with high prevalence of advanced liver fibrosis which coexists with a mixture of elevated plasma inflammation and microbial translocation biomarkers. The significant associations of advanced liver fibrosis with FGF-basic and SCD14 may reveal pathogenic mechanisms and potential clinical intervention targets of liver fibrosis in HCV/HIV coinfection.

\section{Abbreviations}

HIV: Human Immunodeficiency Virus $\triangle H C V$ : hepatitis $C$ virus; cART: Combination active antiretroviral therapy; FIB-4: Fibrosis Index Based on the 4 Factor; OR: Odds Ratio; 95\% Cl: 95\% Confidence Interval

\section{Declarations}

\section{Acknowledgements}

We would like to thank all participants of the study. We thank Dr. Emily Kestenbaum from Medical School for International Health at Ben Gurion University of Israel for editorial assistance.

\section{Author's contributions}

The study was conceived and supervised by NH and SD. RT, SZ, RY, YY, JW and SY conducted field survey, data collection and laboratory testing. $\mathrm{XC}$ analyzed the data and drafted the manuscript. $\mathrm{XL}$ and $\mathrm{NH}$ critically reviewed and revised the manuscript. All authors approved the final manuscript.

\section{Funding}

This work was supported by the Natural Science Foundation of China (Grant No. 81773485; 81373062), the National Science and Technology Major Projects on Infectious Diseases of China (Grant No. 
2018ZX10721102-004), and Shanghai Municipal Health Commission (Grant No. GWTD2015S05).

\section{Availability of supporting data}

Not applicable

\section{Ethics approval and consent to participate}

The study was approved by the Institutional Review Board of Fudan University School of Public Health, Shanghai, China. All subjects provided informed consent prior to enrollment.

\section{Consent for publication}

Not applicable

\section{Competing interests}

None

\section{References}

1. Platt L, Easterbrook P, Gower E, McDonald B, Sabin K, McGowan C, et al. Prevalence and burden of $\mathrm{HCV}$ co-infection in people living with HIV: a global systematic review and meta-analysis. Lancet Infect Dis 2016; 16(7):797-808.

2. Lo Re V, 3rd, Kallan MJ, Tate JP, Localio AR, Lim JK, Goetz MB, et al. Hepatic decompensation in antiretroviral-treated patients co-infected with HIV and hepatitis $C$ virus compared with hepatitis $C$ virus-monoinfected patients: a cohort study. Ann Intern Med 2014; 160(6):369-379.

3. Bao Y, Larney S, Peacock A, Colledge S, Grebely J, Hickman M, et al. Prevalence of HIV, HCV and HBV infection and sociodemographic characteristics of people who inject drugs in China: A systematic review and meta-analysis. Int J Drug Policy 2019; 70:87-93.

4. Chen ZW, Li Z, Wang QH, Wu XL, Li H, Ren H, et al. Large Disparity between Prevalence and Treatment Rates for Hepatitis C in Western China. J Clin Trans/ Hepatol 2018; 6(4):385-390.

5. Page EE, Nelson M, Kelleher P. HIV and hepatitis $C$ coinfection: pathogenesis and microbial translocation. Curr Opin HIV AIDS 2011; 6(6):472-477.

6. Bruno R, Galastri S, Sacchi P, Cima S, Caligiuri A, DeFranco R, et al. gp120 modulates the biology of human hepatic stellate cells: a link between HIV infection and liver fibrogenesis. Gut 2010; 59(4):513520.

7. Bruno R, Sacchi P, Puoti M, Maiocchi L, Patruno SF, Cima S, et al. Pathogenesis of liver damage in HCV-HIV patients. AIDS Rev 2008; 10(1):15-24.

8. Kim AY, Chung RT. Coinfection with HIV-1 and HCV-a one-two punch. Gastroenterology 2009; 137(3):795-814. 
9. Dinh DM, Volpe GE, Duffalo C, Bhalchandra S, Tai AK, Kane AV, et al. Intestinal microbiota, microbial translocation, and systemic inflammation in chronic HIV infection. J Infect Dis 2015; 211(1):19-27.

10. Albillos A, Lario M, Alvarez-Mon M. Cirrhosis-associated immune dysfunction: distinctive features and clinical relevance. J Hepatol 2014; 61(6):1385-1396.

11. Nakamoto $\mathrm{N}$, Kanai T. Role of toll-like receptors in immune activation and tolerance in the liver. Front Immunol 2014; 5:221.

12. Sacchi P, Cima S, Zuccaro V, Columpsi P, Sarda C, Mariani M, et al. Understanding the Mechanisms of Fibrogenesis in HIV/HCV-Coinfected Patients: Implications for Clinical Practice. AIDS Rev 2015; 17(3):159-170.

13. Sitia G, De Bona A, Bagaglio S, Galli L, Paties CT, Uberti-Foppa C, et al. Naive HIV/HCV-coinfected patients have higher intrahepatic pro-inflammatory cytokines than coinfected patients treated with antiretroviral therapy. Antivir Ther 2006; 11(3):385-389.

14. Hunt PW, Lee SA, Siedner MJ. Immunologic Biomarkers, Morbidity, and Mortality in Treated HIV Infection. J Infect Dis 2016; 214 Suppl 2:S44-50.

15. Duan S, Jin Z, Liu X, Yang Y, Ye R, Tang R, et al. Tobacco and alcohol use among drug users receiving methadone maintenance treatment: a cross-sectional study in a rural prefecture of Yunnan Province, Southwest China. BMJ Open 2017; 7(3):e014643.

16. Ding Y, Duan S, Ye R, Yang Y, Yao S, Wang J, et al. More improvement than progression of liver fibrosis following antiretroviral therapy in a longitudinal cohort of HIV-infected patients with or without HBV and HCV co-infections. J Viral Hepat 2017; 24(5):412-420.

17. Tada T, Kumada T, Toyoda H, Kiriyama S, Tanikawa M, Hisanaga $Y$, et al. Long-term prognosis of patients with chronic hepatitis $\mathrm{C}$ who did not receive interferon-based therapy: causes of death and analysis based on the FIB-4 index. J Gastroenterol 2016; 51(4):380-389.

18. Sterling RK, Lissen E, Clumeck N, Sola R, Correa MC, Montaner J, et al. Development of a simple noninvasive index to predict significant fibrosis in patients with HIV/HCV coinfection. Hepatology 2006; 43(6):1317-1325.

19. Zhang T, Tully DC, Zhou S, He N. Characteristics of HCV co-infection among HIV infected individuals from an area with high risk of blood-borne infections in central China. PLoS One 2014; 9(4):e94219.

20. Chen X, Liu X, Tang R, Ye R, Yang Y, Yao S, et al. Fibrosis-4 index predicts mortality in HIV/HCV coinfected patients receiving combination antiretroviral therapy in rural China. Biosci Trends 2019; 13(1):32-39.

21. Zhu W, Mao Y, Tang H, McGoogan JM, Zhang ZF, Detels R, et al. Spectrum of malignancies among the population of adults living with HIV infection in China: A nationwide follow-up study, 2008-2011. PLoS One 2019; 14(7):e0219766.

22. Down C, Mehta N, Marks K. The Risk of Cardiovascular Disease, Diabetes, Liver-Related Outcomes, and Death Over 10 Years in HIV/HCV-Coinfected Patients With and Without Steatosis. AIDS Res Hum Retroviruses 2016; 32(9):868-871. 
23. Merli M, Galli L, Castagna A, Salpietro S, Gianotti N, Messina E, et al. Diagnostic accuracy of APRI, FIB-4 and Forns for the detection of liver cirrhosis in HIV/HCV-coinfected patients. New Microbiol 2016; 39(2):110-113.

24. Pembroke T, Deschenes M, Lebouche B, Benmassaoud A, Sewitch M, Ghali P, et al. Hepatic steatosis progresses faster in HIV mono-infected than HIV/HCV co-infected patients and is associated with liver fibrosis. J Hepatol 2017; 67(4):801-808.

25. Luo X, Duan S, Duan Q, Pu Y, Yang Y, Ding Y, et al. Alcohol use and subsequent sex among HIVinfected patients in an ethnic minority area of Yunnan Province, China. PLoS One 2013; 8(4):e61660.

26. Shmagel KV, Saidakova EV, Shmagel NG, Korolevskaya LB, Chereshnev VA, Robinson J, et al. Systemic inflammation and liver damage in HIV/hepatitis C virus coinfection. HIV medicine 2016; 17(8):581-589.

27. Funderburg NT, Andrade A, Chan ES, Rosenkranz SL, Lu D, Clagett B, et al. Dynamics of immune reconstitution and activation markers in HIV+ treatment-naive patients treated with raltegravir, tenofovir disoproxil fumarate and emtricitabine. PLoS One 2013; 8(12):e83514.

28. Das JR, Gutkind JS, Ray PE. Circulating Fibroblast Growth Factor-2, HIV-Tat, and Vascular Endothelial Cell Growth Factor-A in HIV-Infected Children with Renal Disease Activate Rho-A and Src in Cultured Renal Endothelial Cells. PLoS One 2016; 11(4):e0153837.

29. Keating SM, Dodge JL, Norris PJ, Heitman J, Gange SJ, French AL, et al. The effect of HIV infection and HCV viremia on inflammatory mediators and hepatic injury-The Women's Interagency HIV Study. PLoS One 2017; 12(9):e0181004.

30. McGaha TL, Bona CA. Role of profibrogenic cytokines secreted by T cells in fibrotic processes in scleroderma. Autoimmun Rev 2002; 1(3):174-181.

31. Kaspar MB, Sterling RK. Mechanisms of liver disease in patients infected with HIV. BMJ Open Gastroenterol 2017; 4(1):e000166.

32. Knight B, Lim R, Yeoh GC, Olynyk JK. Interferon-gamma exacerbates liver damage, the hepatic progenitor cell response and fibrosis in a mouse model of chronic liver injury. $J$ Hepatol 2007; 47(6):826-833.

33. Kubota A, Okamura S, Omori F, Shimoda K, Otsuka T, Ishibashi H, et al. High serum levels of granulocyte-macrophage colony-stimulating factor in patients with liver cirrhosis and granulocytopenia. Clin Lab Haematol 1995; 17(1):61-63.

34. Balagopal A, Philp FH, Astemborski J, Block TM, Mehta A, Long R, et al. Human immunodeficiency virus-related microbial translocation and progression of hepatitis C. Gastroenterology 2008; 135(1):226-233.

35. Graham CS, Wells A, Liu T, Sherman KE, Peters M, Chung RT, et al. Antigen-specific immune responses and liver histology in HIV and hepatitis C coinfection. AIDS 2005; 19(8):767-773.

36. de Lalla C, Galli G, Aldrighetti L, Romeo R, Mariani M, Monno A, et al. Production of profibrotic cytokines by invariant NKT cells characterizes cirrhosis progression in chronic viral hepatitis. $J$ Immunol 2004; 173(2):1417-1425. 
37. Jain MK, Adams-Huet B, Terekhova D, Kushner LE, Bedimo R, Li X, et al. Acute and chronic immune biomarker changes during interferon/ribavirin treatment in HIV/HCV co-infected patients. $J$ Viral Hepat 2015; 22(1):25-36.

38. Zhou Z, Xu MJ, Cai Y, Wang W, Jiang JX, Varga ZV, et al. Neutrophil-Hepatic Stellate Cell Interactions Promote Fibrosis in Experimental Steatohepatitis. Cell Mol Gastroenterol Hepatol 2018; 5(3):399-413.

39. Balagopal A, Gama L, Franco V, Russell JN, Quinn J, Higgins Y, et al. Detection of microbial translocation in HIV and SIV infection using the Limulus amebocyte lysate assay is masked by serum and plasma. PLoS One 2012; 7(8):e41258.

40. Balagopal A, Gama L, Franco V, Russell JN, Quinn J, Higgins Y, et al. Detection of microbial translocation in HIV and SIV infection using the Limulus amebocyte lysate assay is masked by serum and plasma. 2012; 7(8):e41258.

41. French AL, Evans CT, Agniel DM, Cohen MH, Peters M, Landay AL, et al. Microbial translocation and liver disease progression in women coinfected with HIV and hepatitis C virus. J Infect Dis 2013; 208(4):679-689.

42. Negi N, Singh R, Sharma A, Das BK, Vajpayee M. Comparative evaluation of microbial translocation products (LPS, sCD14, IgM Endocab) in HIV-1 infected Indian individuals. Microb Pathog 2017; 111:331-337.

\section{Figures}




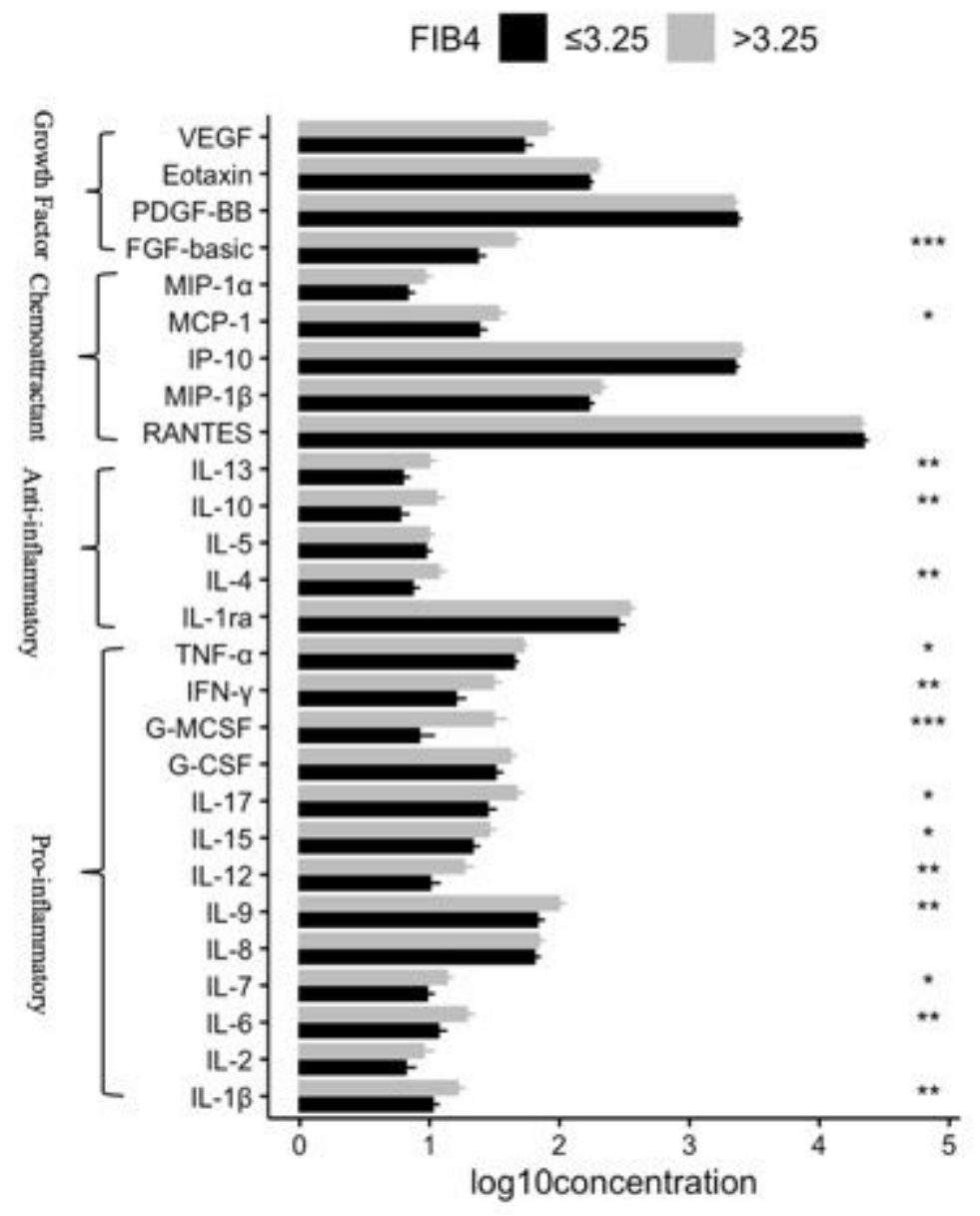

Figure 1

Comparison of log10 transformed plasma concentration of tested biomarkers between participants with different FIB-4 score. ${ }^{*} p<0.05,{ }^{*} p<0.01$, and ${ }^{* *}{ }^{*} p<0.001$. All the $p$-values were calculated by MannWhitney tests. 


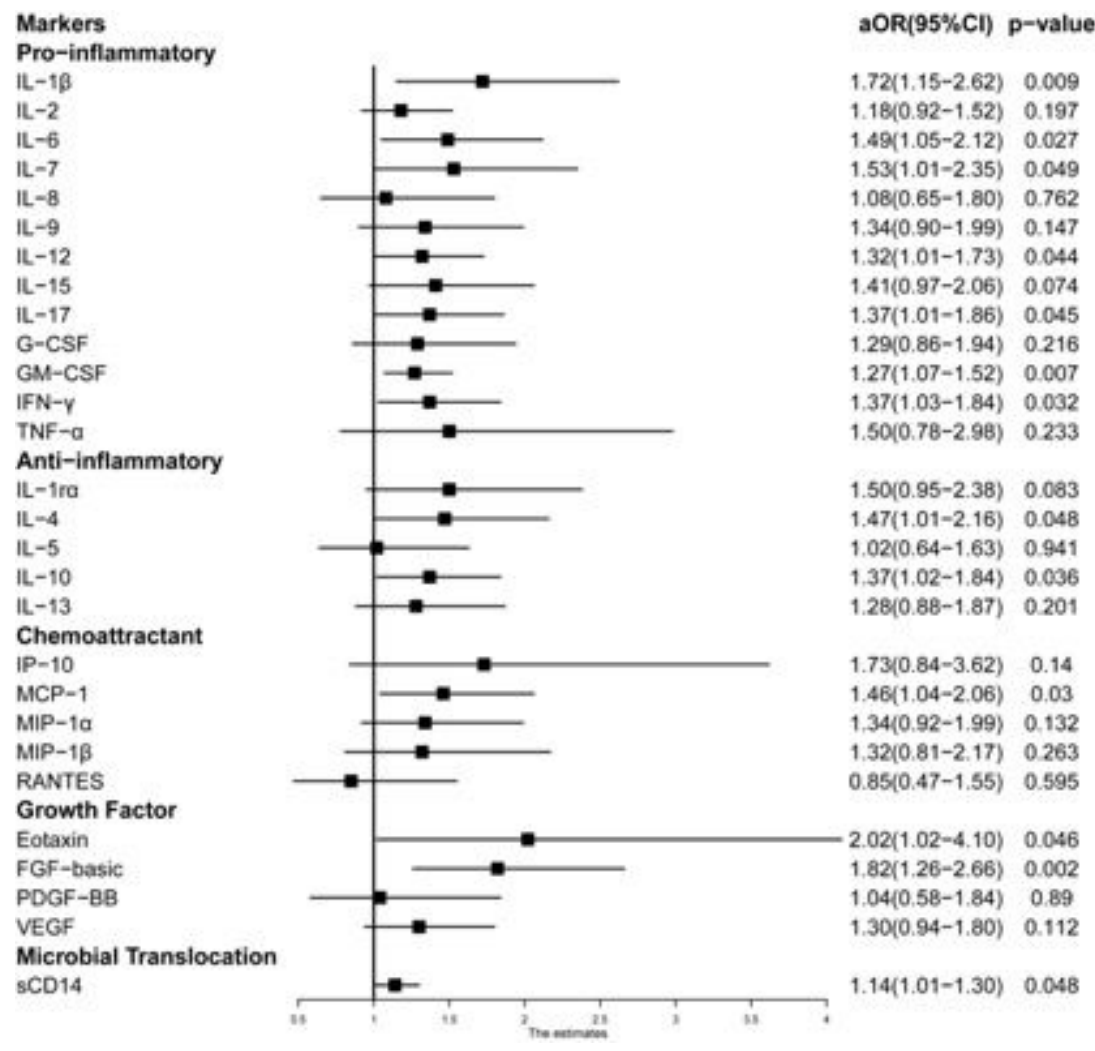

Figure 3

Adjusted odds ratios and $95 \%$ confidence intervals for the associations between plasma cytokine levels (log10 transformed) and advanced liver fibrosis among HIV/HCV coinfected patients. Each association was assessed in a separate multivariable logistic regression model adjusting for age, sex, ethnicity, current HIV RNA, current HCV RNA, current CD4 count, years on CART, and ART regimen.

\section{Supplementary Files}

This is a list of supplementary files associated with this preprint. Click to download.

- supplementary20200329.docx

- supplementary20200329.docx 